\title{
Case Report \\ Jejunojejunal Intussusception Induced by a Gastrointestinal Stromal Tumor
}

\begin{abstract}
Ali H. Zakaria and Salam Daradkeh
Istishari Hospital, The University of Jordan, P.O. Box 13261, Amman 11942, Jordan

Correspondence should be addressed to Salam Daradkeh, daradkeh@ju.edu.jo

Received 10 October 2012; Accepted 25 October 2012

Academic Editors: S.-i. Kosugi and M. Nikfarjam

Copyright () 2012 A. H. Zakaria and S. Daradkeh. This is an open access article distributed under the Creative Commons Attribution License, which permits unrestricted use, distribution, and reproduction in any medium, provided the original work is properly cited.

Background. Adult intussusception is a rare entity representing less than $1 \%$ of all intestinal obstructions. Diagnosis of the condition is difficult requiring a high index of suspicion and the utilization of imaging studies, especially CT scans. Diagnostic laparoscopy and/or exploratory laparotomy can be used as a diagnostic and therapeutic intervention. In over $90 \%$ of cases, an underlying lead point is identified. In the patient described here, it was a gastrointestinal stromal tumor (GIST), a relatively rare mesenchymal tumor comprising only $0.2-1.0 \%$ of the gastrointestinal tract neoplasms and believed to originate from neoplastic transformation of the interstitial cells of Cajal. GISTs may occur anywhere along the gastrointestinal tract, but most commonly arise in the stomach and small intestine. Literature review revealed only few cases reporting GISTs as a leading point of adult's intussusception. Case Presentation. In this report, we are presenting a rare case of jejunojejunal intussusception in a 78-year-old female patient with a GIST located in the terminal jejunum being the leading point, demonstrating the importance of imaging studies, especially CT scan, laparoscopy, and exploratory laparotomy as diagnostic and therapeutic interventions.
\end{abstract}

\section{Introduction}

Intestinal invagination or intussusception is the leading cause of intestinal obstruction in children, but it is an uncommon process in adults, accounting for only $5 \%$ of all intussusceptions and 1\% of all intestinal obstruction. Unlike childhood intussusception which is idiopathic in $90 \%$ of cases, $70-90 \%$ of adult cases have a demonstrable lead point, with a well-definable neoplastic abnormality being the etiology in $65 \%$ of cases $[1,2]$.

Adult intussusception may present with acute, subacute, or chronic nonspecific symptoms. Therefore, the initial diagnosis often is missed or delayed till the patient is in the operating room. There is a surgical consensus that adult intussusception requires surgical resection because the majority of patients have intraluminal lesions. However, there is controversy about the need for reduction of the intussusception and the extent of resection to be performed [2].

\section{Case Report}

A seventy-eight-year-old female patient with a previous medical history of diabetes mellitus, hypertension, ischemic heart disease, and Hypothyroidism presented with a oneweek history of abdominal pain and nausea with vomiting following meals. Her symptoms progressively worsened to severe abdominal distention, anorexia and obstipation on the day of admission. She had no fever, chills, bleeding per rectum, or previous abdominal surgeries. Four weeks earlier the patient had similar symptoms. A plain abdominal X-ray and CT scan which were done at that time showed dilated small bowel loop in right lower quadrant without detected lesions or significant wall thickening, and she had been treated conservatively. After resolution of her symptoms-during the previous episode-an upper and lower GI endoscopy were done and revealed moderate erosive esophagitis, Helicobacter pylori positive gastritis and duodenitis, and an essentially normal colonic exam. 
Her vital signs on admission were within normal limits. Examination showed a distended abdomen with hyperactive bowel sounds and generalized tenderness without peritoneal signs. Her laboratory investigations showed anemia with hemoglobin of $7.2 \mathrm{~g} / \mathrm{dL}$ and white cell count of $9.9 \times$ $10^{3} / \mathrm{mm}^{3}$. Abdominal X-ray revealed dilated proximal bowel loops with multiple air fluid levels. Conservative measures with bowel rest, nasogastric intubation, and intravenous fluids failed to control her symptoms. Abdominal and pelvic CT-scan showed markedly dilated fluid filled small bowel loops with one loop showing central hyperdense area, and a thick soft tissue mass around it with multiple small hypodense areas, referred to as the target sign [3] (Figure 1).

Diagnostic laparoscopy which revealed a distal jejunojejunal intussusception was followed by limited laparotomy and resection then anastomosis was performed (Figure 2). The leading point of the invagination was jejunal tumor (Figure 3). Histopathologic examination of the resected specimen revealed a GIST of intermediate risk, and resection was complete with viable ends.

The patient had an uneventful postoperative recovery and was discharged in a well condition. She was doing well on subsequent clinic followup.

\section{Discussion}

Adult intussusception is an uncommon clinical entity encountered by surgeons. The exact mechanism is unknown, and it is believed that any lesion in the bowel wall or irritant within the lumen that alters normal peristaltic activity is able to initiate invagination. It is most commonly located at the junctions between freely moving segments and retroperitoneally or adhesionally fixed segments [4].

About $90 \%$ of occurrences in adults have a well-defined pathological lead point, which may be a benign—such as benign neoplasms, inflammatory lesions, Meckel's diverticuli, appendix, and adhesions_-or malignant lesion. In small intestine, malignant lesions (either primary or metastatic) account for $14-47 \%$ of cases, while malignant etiology is more prominent in large bowel representing up to $66 \%$ of the cases [2].

Most adult patients with intussusception present with chronic and nonspecific symptoms suggestive of intestinal obstruction. Abdominal pain is the most common symptom followed by nausea, vomiting, and a palpable abdominal mass $[1,2]$.

Preoperative imaging may help in identifying the causative lesion. Plain abdominal X-rays are typically the first diagnostic tool; with barium studies (showing "stacked coin" or "coiled spring" in upper GI series and "cup-shaped defect" in barium enema), ultrasonography (showing "target and doughnut sign" on transverse view and the "pseudokidney sign" in longitudinal view), and colonoscopy are also useful tools for evaluating intussusception [5-7].

In recent years, with a diagnostic accuracy of $58-100 \%$ in recent series, abdominal CT-scan (with the characteristic "target sign") has been reported to be the most useful tool for diagnosis of intestinal intussusception and is regarded superior to the above mentioned studies $[3,8,9]$.

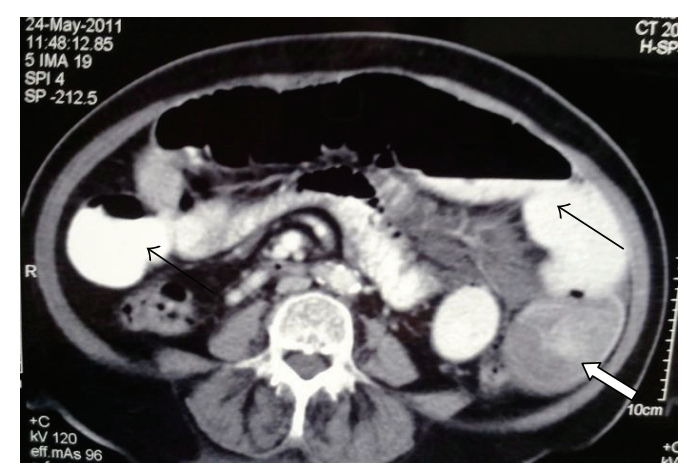

FIGURE 1: Computed tomography of abdomen showing dilated bowel loops (arrows) and the target sign of intussusception (white arrow).

Treatment of adult intussusception is always surgical. However, optimal management remains controversial. Most of the debate focuses on the issue of primary resection versus initial reduction followed by a more limited resection $[2,9]$, keeping in mind that reduction should not be attempted with any degree of suspicion of malignancy, due to possible risks of intraluminal seeding, venous embolization in regions of ulcerated mucosa, and anastomotic complications, which may potentially lead to bowel perforation [10]. Recently, there are several case reports about using laparoscopy as a minimally invasive technique for both diagnosis and treatment of adult intussusceptions.

Mesenchymal tumors constitute only $1 \%$ of primary GI cancers with GISTs being the most common. The annual incidence is between 7 and 20 cases per million per year [11]. It occurs predominantly in middle-aged and older individuals and rarely in those under the age of 40 . The majority of cases are sporadic; however, several familial cases with heritable mutations in the KIT gene have been identified [12].

GISTs are thought to derive from neoplastic transformation of interstitial cells of Cajal (ICC). They may occur throughout the GI tract from the esophagus to the anus, but most commonly are found in the stomach $(40-60 \%)$ and jejunum/ileum (25-30\%) [12]. Duodenum (5\%), colorectum $(5-15 \%)$, and esophagus $(\leq 1 \%)$ are less common sites.

Sometimes GISTs are asymptomatic and are discovered incidentally during an endoscopy or on a CT done for another purpose. More often, they are associated with nonspecific symptoms (i.e., early satiety, bloating) unless complicated with ulceration and overt GI bleeding (40\% of the cases) or grow large enough to cause pain, mass or a lead point of intussusception and intestinal obstruction $(20 \%$ of the cases) [12].

Contrast-enhanced CT is a preferred initial imaging study for screening and staging. However, other procedures such as ultrasound, endoscopy, intestinal capsule, and PET scan may also be used.

Surgical "en bloc" segmental resection with the goal of achieving negative resection margins is the treatment of choice for potentially resectable tumors, while initial therapy 

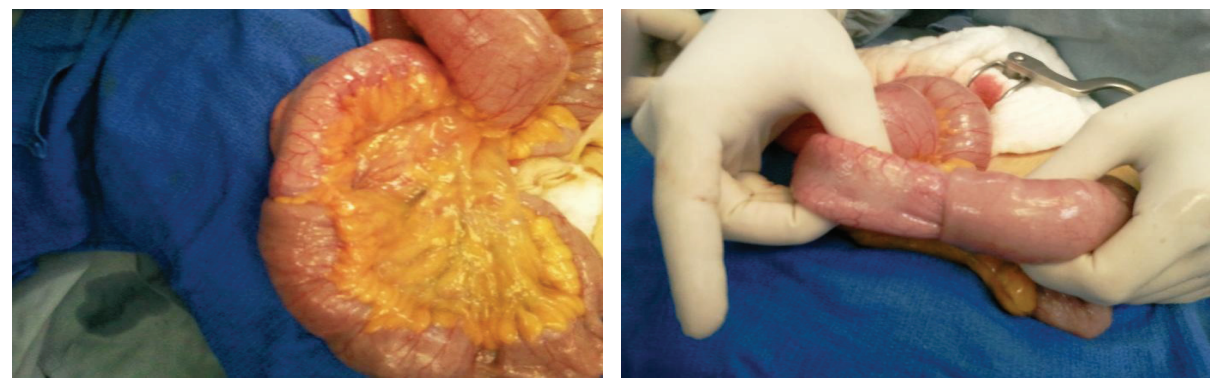

FIGURE 2: Intraoperative view showing the jejunojejunal intussusception.

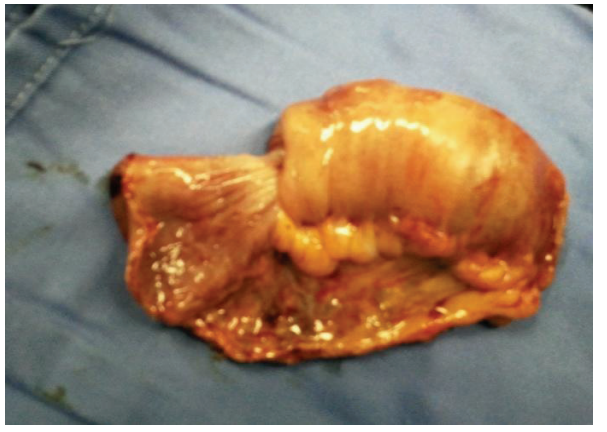

FIgURE 3: Jejunal tumor (GIST) was the leading point of intussusception.

with imatinib may be preferred if a tumor is borderline resectable, or if resection would necessitate extensive organ disruption.

The prognosis of small intestine GISTs depends upon the adequacy of resection, tumor size, mitotic activity, and location within the small bowel, with small intestine having a worse prognosis than stomach [13-15].

\section{Conclusion}

Intussusception in adults occurs relatively rarely; however, in $90 \%$ of cases a specific lead point is identified. The diagnosis may be challenging because of nonspecific symptoms, and sometimes it is impossible to reach the diagnosis of intussusception as a cause of obstruction until laparotomy is done. CT-scan is the most useful imaging modality in diagnosis. An underlying malignant lesion might be the lead point especially in large bowel. Therefore, surgeons should think of intussusception as a cause of intestinal obstruction, and they should be familiar with the various treatment options. The decision whether to undertake resection or reduction followed by resection is case specific, and it should be tailored according to the situation.

\section{References}

[1] T. Azar and D. L. Berger, "Adult intussusception," Annals of Surgery, vol. 226, pp. 134-138, 1997.

[2] D. G. Begos, A. Sandor, and I. M. Modlin, "The diagnosis and management of adult intussusception," American Journal of Surgery, vol. 173, no. 2, pp. 88-94, 1997.
[3] G. Gayer, S. Apter, C. Hofmann et al., "Intussusception in adults: CT diagnosis," Clinical Radiology, vol. 53, no. 1, pp. 53$57,1998$.

[4] L. T. Wang, C. C. Wu, J. C. Yu, C. W. Hsiao, C. C. Hsu, and S. W. Jao, "Clinical entity and treatment strategies for adult intussusceptions: 20 years' experience," Diseases of the Colon and Rectum, vol. 50, no. 11, pp. 1941-1949, 2007.

[5] L. K. Eisen, J. D. Cunningham, and A. H. Aufses Jr., "Intussusception in adults: institutional review," Journal of the American College of Surgeons, vol. 188, no. 4, pp. 390-395, 1999.

[6] D. L. Weissberg, W. Scheible, and G. R. Leopold, "Ultrasonographic appearance of adult intussusception," Radiology, vol. 124, no. 3, pp. 791-792, 1977.

[7] L. M. Hurwitz and S. L. Gertler, "Colonoscopic diagnosis of ileocolic intussusception," Gastrointestinal Endoscopy, vol. 32, no. 3, pp. 217-218, 1986.

[8] J. Bar-Ziv and A. Solomon, "Computed tomography in adult intussusception," Gastrointestinal Radiology, vol. 16, no. 3, pp. 264-266, 1991.

[9] M. Barussaud, N. Regenet, X. Briennon et al., "Clinical spectrum and surgical approach of adult intussusceptions: a multicentric study," International Journal of Colorectal Disease, vol. 21, no. 8, pp. 834-839, 2006.

[10] H. Yamada, T. Morita, M. Fujita, Y. Miyasaka, N. Senmaru, and T. Oshikiri, "Adult intussusception due to enteric neoplasms," Digestive Diseases and Sciences, vol. 52, no. 3, pp. 764-766, 2007.

[11] T. Tran, J. A. Davila, and H. B. El-Serag, "The epidemiology of malignant gastrointestinal stromal tumors: an analysis of 1,458 cases from 1992 to 2000," American Journal of Gastroenterology, vol. 100, no. 1, pp. 162-168, 2005.

[12] M. Miettinen and J. Lasota, "Gastrointestinal stromal tumors-definition, clinical, histological, immunohistochemical, and molecular genetic features and differential diagnosis," Virchows Archiv, vol. 438, no. 1, pp. 1-12, 2001.

[13] R. P. DeMatteo, J. J. Lewis, D. Leung, S. S. Mudan, J. M. Woodruff, and M. F. Brennan, "Two hundred gastrointestinal stromal tumors: recurrence patterns and prognostic factors for survival," Annals of Surgery, vol. 231, no. 1, pp. 51-58, 2000.

[14] W. L. Yang, J. R. Yu, Y. J. Wu et al., “Duodenal gastrointestinal stromal tumor: clinical, pathologic, immunohistochemical characteristics, and surgical prognosis," Journal of Surgical Oncology, vol. 100, no. 7, pp. 606-610, 2009.

[15] M. Miettinen, H. Makhlouf, L. H. Sobin, and J. Lasota, "Gastrointestinal stromal tumors of the jejunum and ileum: a clinicopathologic, immunohistochemical, and molecular genetic study of 906 cases before imatinib with long-term follow-up," American Journal of Surgical Pathology, vol. 30, no. 4, pp. 477489, 2006. 


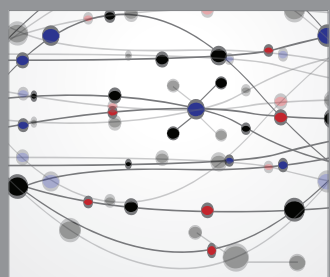

The Scientific World Journal
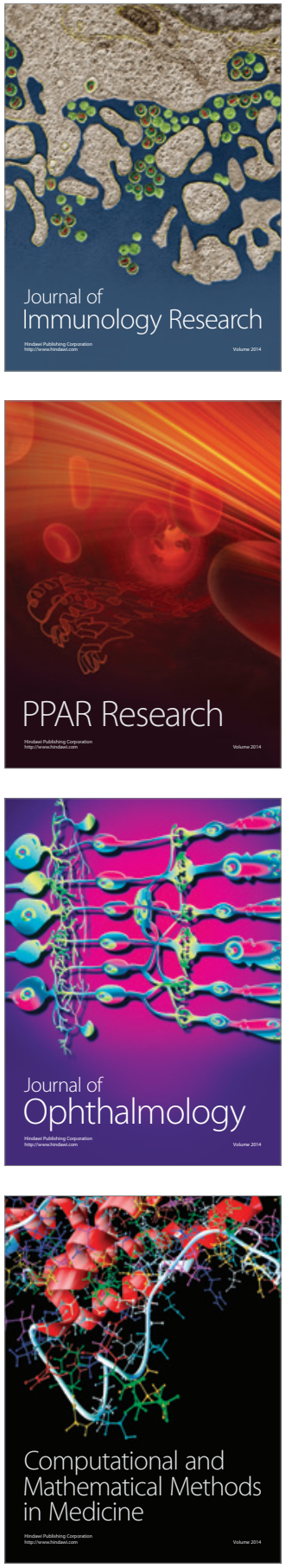

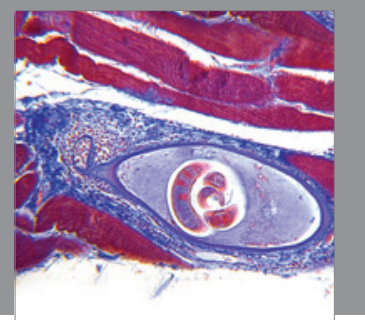

Gastroenterology

Research and Practice
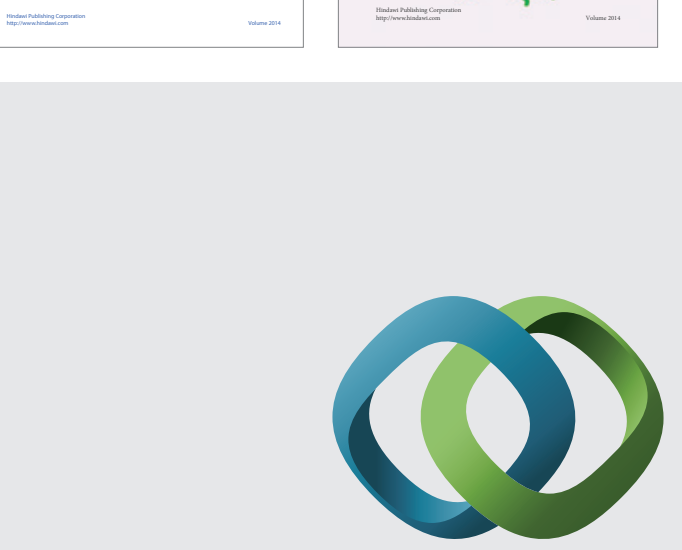

\section{Hindawi}

Submit your manuscripts at

http://www.hindawi.com
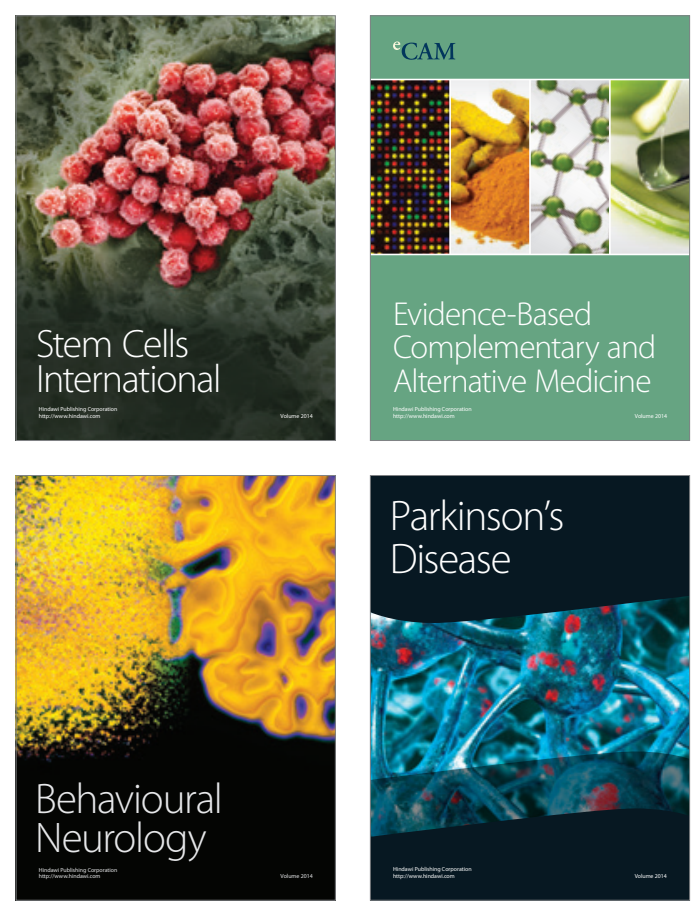

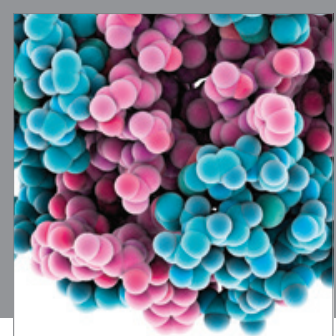

Journal of
Diabetes Research

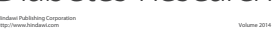

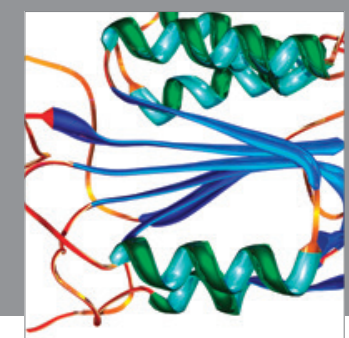

Disease Markers
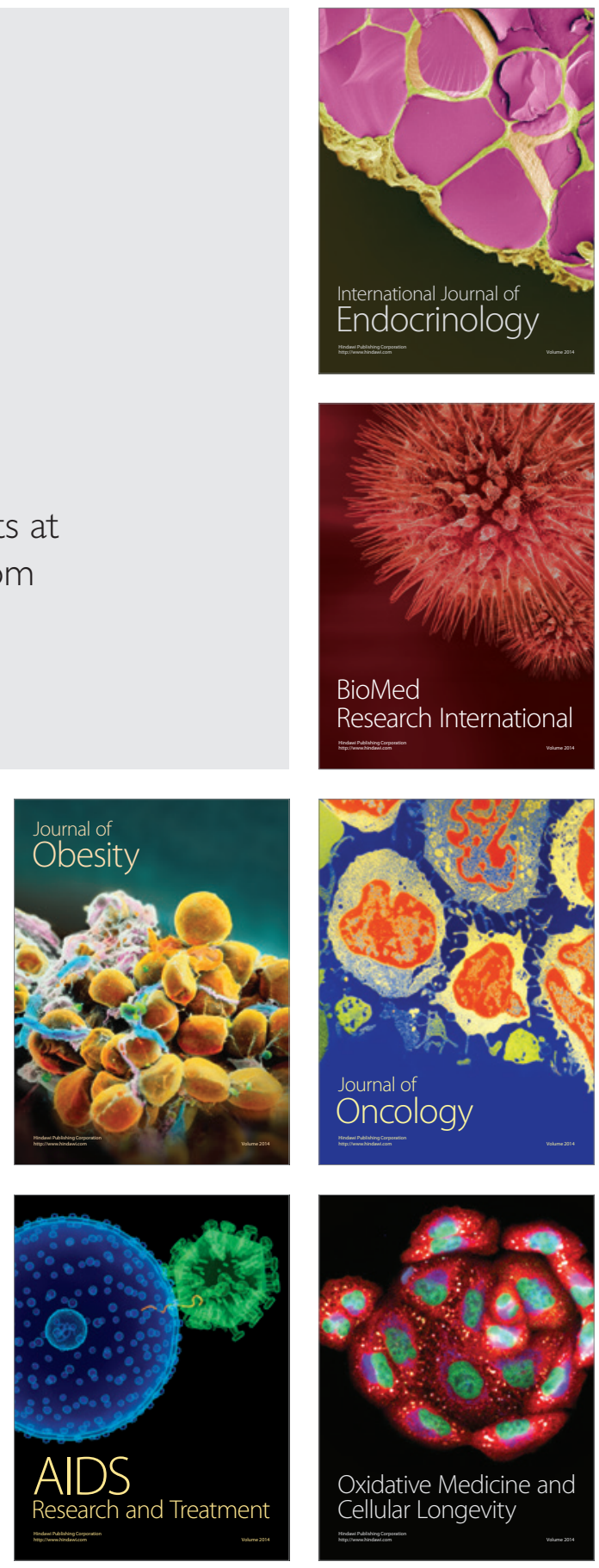